\title{
Forty Years of Chromatographia: A Scientific Review
}

In its first year of publication in 1968 Chromatographia started with a total of 103 papers. As usual at that time, the subject of the papers was mainly gas chromatography with a few papers on liquid chromatography, particularly thin layer chromatography. Some of these 103 papers were published in German and French, but most of the papers were in English, despite the fact that the publishers offered to accept papers in all three languages. Ten years later all papers were published in English (with the language "polished" for nonnative English speaking authors). The renaissance of liquid chromatography in the 1970s led to a greater balance between the papers in gas and liquid chromatography. Nowadays around $15 \%$ of the papers have gas chromatography and its applications as their subject.

Originally fundamental and theoretical aspects of chromatography were the main content of the papers. As chromatographic techniques became more and more mature, the broad fields of application of chromatography in chemical, environmental and pharmaceutical sciences have been represented in around 5,000 full size (Original) papers

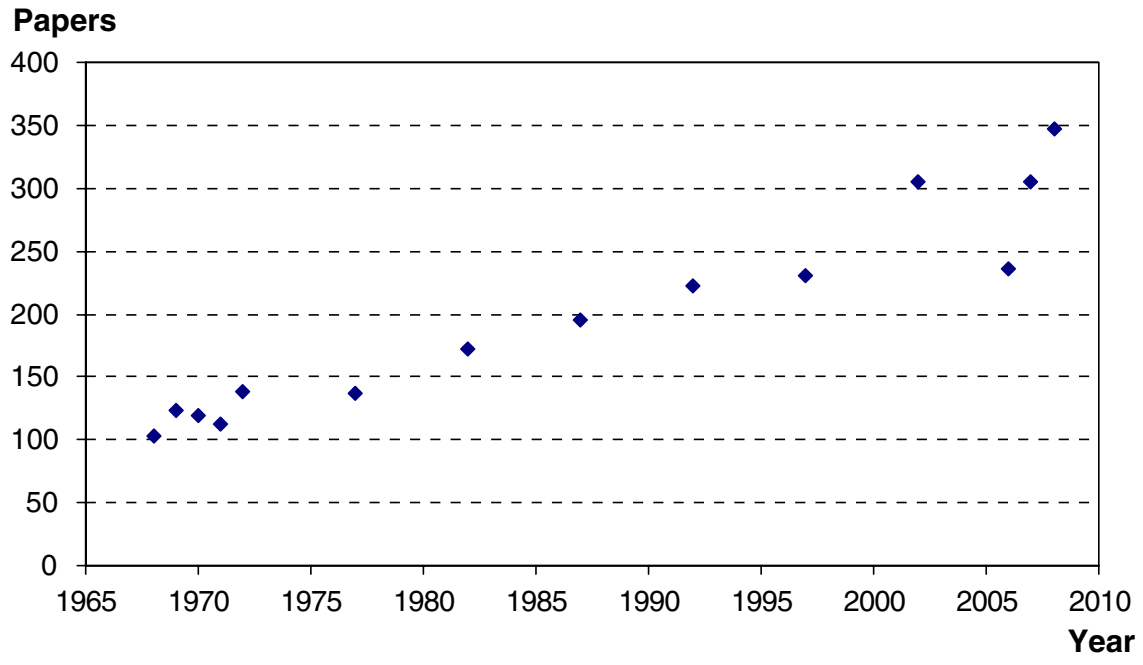

and over 1,000 Short Communications mainly from the USA and Europe.

The figure shows the steady increase of papers during these 40 years.

The contributions of emerging countries like China in pharmaceutical science are becoming more and more important and India is represented in the increase of papers submitted in the last 3 years. This indicates that the application of chromatography is becoming a global science.
I am confident that Chromatographia will remain an important scientific journal in the future for the global distribution of knowledge on separation science.

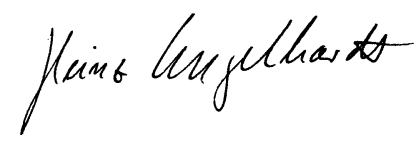

Heinz Engelhardt 